\title{
Shopping System based on RFID Technology
}

\author{
Surya Deo Chaudhary, Alka Singh, Ashutosh Kumar Singh
}

\begin{abstract}
The present system relates to an RFID based shopping system. The system comprises of an RFID reader and various RFID tags are attached to various products. When we go for shopping in supermarkets or malls we select different items according to our choice and put them into a cart and then go to the billing counter for payment, So at the end we get to know about the overall total cost of the products and for sometimes we also have to stand in a queue for a long time resulting in wastage of time for customers. It is needed to develop a system which provides comfort, expediency and efficacy during shopping. The system consists of a Liquid Crystal Display which is mounted on trolley for displaying the information of the selected product and total amount. On 4X4 matrix keypad buyer can set their budget amount for shopping, thus when the total bill amount. If the total bill amount increases than the set value then an alert is generated by the buzzer. After the completion of shopping one can pay the bill by pressing a button wirelessly. Therefore, the system expedites faster billing with reduced time.
\end{abstract}

Index Terms- RFID tag, Microcontroller, RFID reader, $4 X 4$ matrix keypad, LCD

\section{INTRODUCTION}

Shopping malls and Super markets offer regulars all the essential stuffs for example food products, clothes, footwear, and health products etc. at a place. Today, shopping is not only a requirement but also an approach of transformation. Though, the problem which is commonly faced in supermarkets is management of the customers at the billing system. There are huge lines in the supermarkets for paying bill at the counters. By the introduction of RFID technology in the system, system can be advanced and all the problems which exists in manual processing can be overcome by the advanced system. In this RFID based shopping system, RFID tags are connected to the products. When the product having a tag on it comes into the vicinity of the RFID reader during scanning process, then the reader would detect the tag and accordingly the amount of the product and Identity of the product will be stored in the memory. By this, the cost is further added a bill is generated. Therefore the bill would be ready inside the trolley itself. The name of the product, unique id, product cost and overall amount of bill would be displayed. When a button is done pressed after shopping, the bill would be transmitted to the computer present at billing counter. A shopping system based on IOT has been

implemented in [1][2]. Paper [6] discloses the execution of a

Revised Version Manuscript Received on 10 September, 2019

Dr. Surya Deo Chaudhary, Department of Electronics \& Communication Engineering, Noida Institute of Engineering and Technology, Uttar Pradesh, India. (Email: researchnietip@gmail.com)

Alka Singh, Department of Information Technology, Noida Institute of Engineering and Technology, Uttar Pradesh, India. (Email: researchnietip@gmail.com)

Ashutosh Kumar Singh, Department of Electronics \& Communication Engineering, Noida Institute of Engineering and Technology, Uttar Pradesh, India. (Email: researchnietip@gmail.com)
Smart Shopping Cart by the use of Wireless Sensor Networks. The system is reliable as well as cost efficient that also guarantees recognition of deception. Therefore, the system attracts the buyers as well as sellers. Paper [7] talks about an RFID smart shelf which considers custom passive UHF RFID to detect objects having tags with an accuracy. Paper [3] explains an application for smart shopping cart which had been realized using software framework.

Second section of the paper comprises of methodology of the present system. Flow chart of the system is shown in the third section. Then algorithm for the method is explained in section four, experimental results are shown in the next section. Sixth and seventh section of the paper describes the advantages and disadvantages and then followed by the conclusion.

\section{METHODOLOGY}
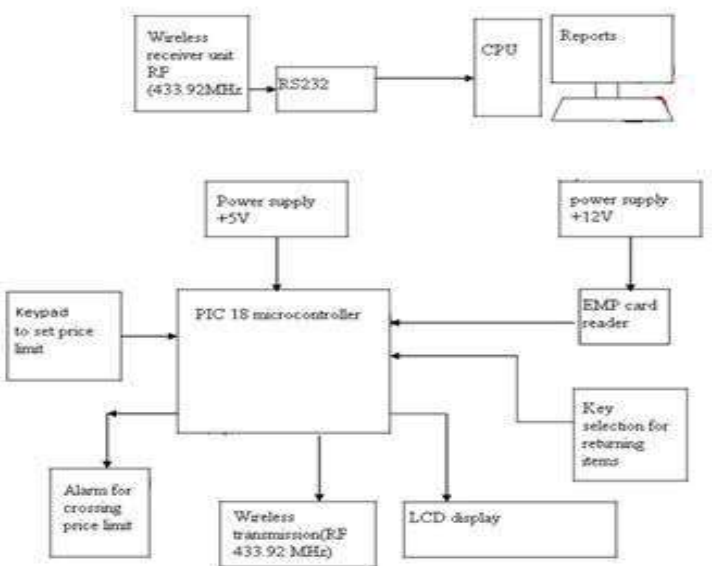

Figure 1 Block diagram of the system

\section{Power Supply}

A step down transformer employed in the system whose input is $230 \mathrm{~V}$ input supply power, $50 \mathrm{HZ}$ and output at the secondary side is 5 Volts. The transformer's output is rectified by a bridge rectifier coupled to the transformer. A 1000 microF capacitor is employed which filters the output obtained from rectifier. A voltage regulator Ic is also used for regulating the output at 5 Volts, the IC is connected to the filter.

\section{Radio Frequency Identification (RFID)}

A high performance RFID proximity readers, having long range of transmission, $12 \mathrm{hkHz}$ frequency and small size. In this technology data transmission between tag and the reader 
takes place by using radio waves. The card is employed for the identification of the item. The tag mainly includes an antenna coil and a silicon chip, and a non-volatile memory. Every item present in the supermarket for sell will have a RFID card on it. An RFID reader is placed in the automated system for scanning the products that are selected and put in the trolley.

\section{RF Transceiver}

An RF transceiver 'CC2500' is used in the system that has low-cost and frequency of $2.4 \mathrm{GHz}$. It's considered for the wireless applications which require very low-power. The circuitry of ' $\mathrm{CC} 2500$ ' is proposed for the frequency range 2450-24903.5 MHz 'ISM' (Industrial, Scientific and Medical) frequency range and 'SRD' (Short Range Device) frequency band. The overall bill of the selected products would be via wireless connection to the computer present in the supermarket by using an RF transceiver.

\section{Serial Communication}

\section{- $R S 232$}

The 'DS14C232' is a low power dual driver/receiver that features an onboard DC to DC converter.

\section{- MAX 232}

A Voltage converter 'MAX 232' is used for linking 'RS232' and microcontroller. The TTL logic level is converted to RS 232 voltage level by MAX 232 in the serial communication. MAX 232 consists of 2 sets of line drivers and receivers for transmitting and receiving the data. $\mathrm{T} 1$ is used to transmit the serial data and $\mathrm{R} 1$ to receive the data. There are requirement of 4 capacitors in MAX 232.

\section{- Buzzer}

The series called 'PS series' consists high-performance and less energy dissipating buzzers. This type of buzzer is employed in the system for notifying the user that the entered price value is less that the amount of total bill.

\section{$\cdot L C D$}

A 16 X 4 LCD is used for display of the product name, product id, product cost and the total bill amount.

\section{-4x4 Matrix Keypad}

This keypad consists of four rows and four columns and offers button states to the microcontroller. The microcontroller is used as a host device in the system. A matrix keypad has been employed in the system which sets the cost limit by the buyer. When the cost limit exceeds the entered value on the keypad then a notification would be sent to the buyer, specifically the notification would be sent by by the buzzer. To make the system simple, there is a function pressing button 2 for long period of time then the entry of item that have been scanned would be removed automatically and equivalent price of the item would be subtracted from the overall amount of bill. For sending the signal to the computer one has to press the button represented by number 1 for long time.

\section{- PIC $18 F 4550$}

The different operations of the microcontroller 'PIC 18 F4550' are storing of scanned data by RFID based system, sending the stored data to the LCD that displays the output, and transmitting the signals to the related output devices.

\section{FLOW DIAGRAM}

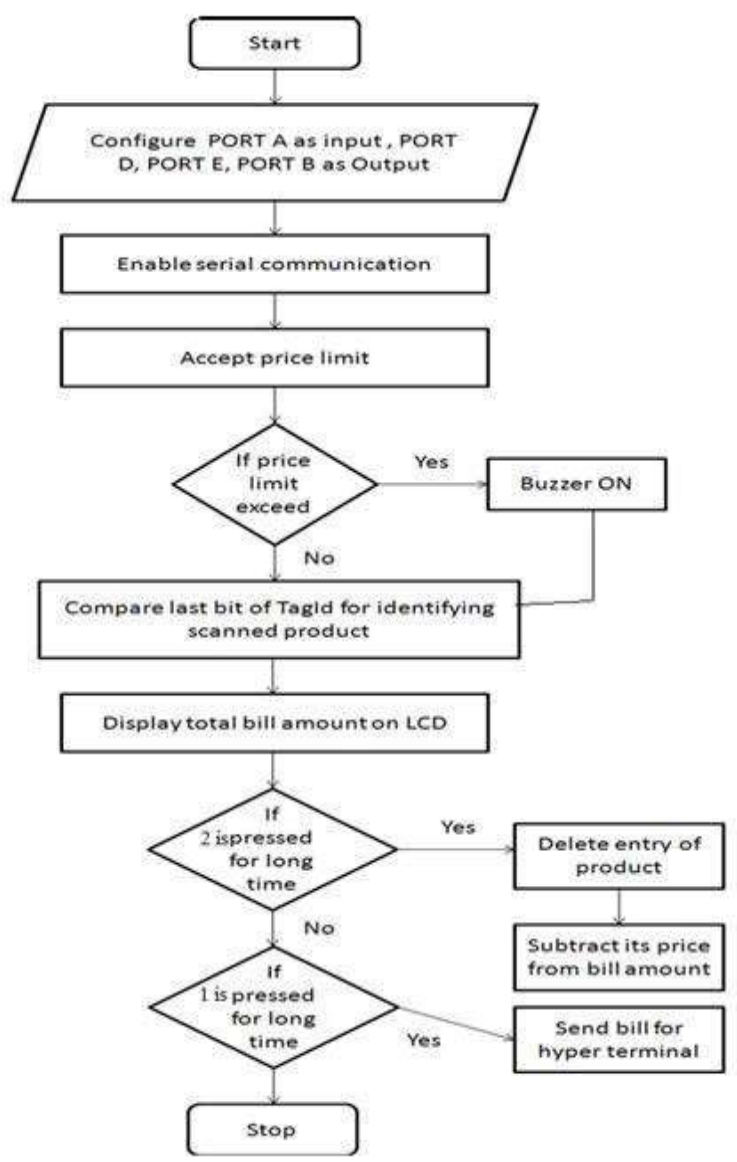

Figure 2 Flow Chart of method

\section{ALGORITHM}

The algorithm for the functioning of system is given as:

$>$ Connecting the supply power to the system.

$>$ Configuring PORT A of host device as an input port and PORT B, E, D as an output port, this is done by doing $\mathrm{C}$ programming.

$>$ Initializing the LCD in the programming.

$>$ Allowing serial communication and transmission of the microcontroller.

$>$ Entering the cost limit by the buyer on $4 \times 4$ matrix keypad.

$>$ Identifying the unique RFID card attached to the item and after this displaying the whole information of that item on the LCD display unit.

$>$ Notifying the user when overall amount of bill exceeds the entered cost limit by turning on a buzzer.

$>$ Deleting the entry of scanned item when the button 2 is pushed for a long duration of time and the amount of that item will be subtracted from the total amount.

$>$ Sending bill to the computer wirelessly when 1 is pushed for long duration of time. 


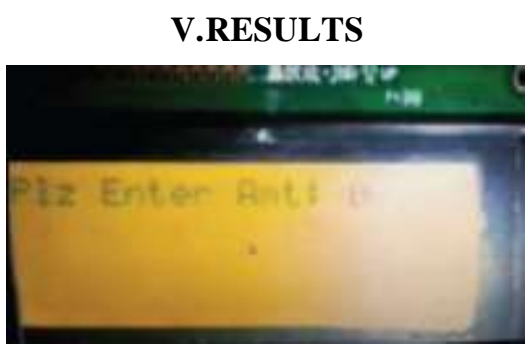

Figure 3 Message for entering amount at LCD

Initially a welcome message will be displayed on the screen after this message a message to enter the cost limit would be shown on the screen and the buyer have to enter the amount by using a keypad.

- The information related to scanned item is displayed as shown below:

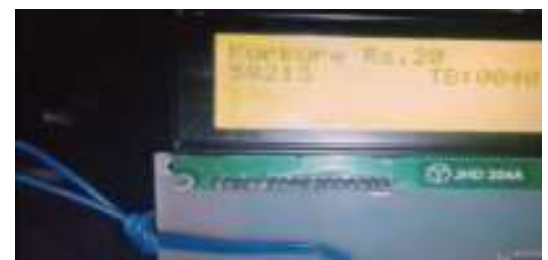

Figure 4 Displayed details

The details of item includes Name of the item, price, unique identity and total amount of bill. Consequently, the item that has been scanned by the buyer named as "Kurkure" costs Rs 20. Overall total amount of bill is calculated by adding of the price of the item to the previous total amount which has been stored in the microcontroller's memory.

The bill displayed on hyper terminal is shown in Figure 5.

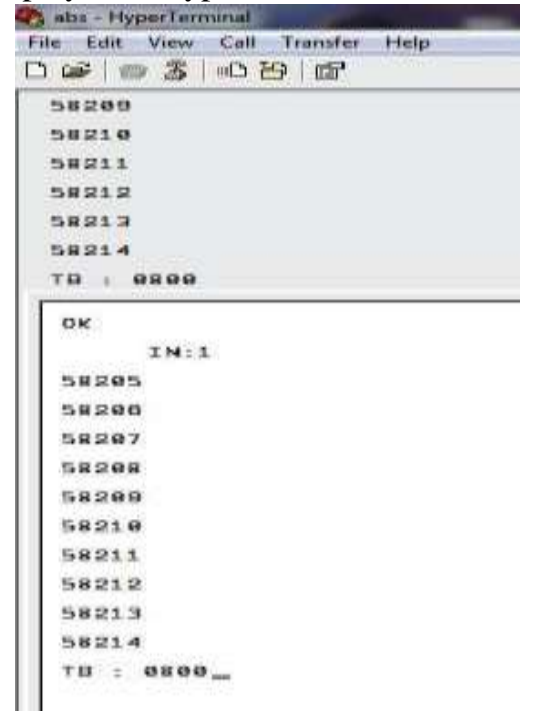

Figure 5 Final bill displayed on hyper terminal

Here 'IN' denotes the input number of trolley. The different numbers coming on the terminal screen are the item's unique ids scanned by reader and TB shows the total amount of bill amount that has to be paid by the buyer at the counter.

Therefore, it can be seen that a customer who is using trolley number 1 has scanned the item having related ids as shown on the screen. The overall total amount of bill is the addition of price of all items, and the buyer has to pay an amount of Rs 800 .

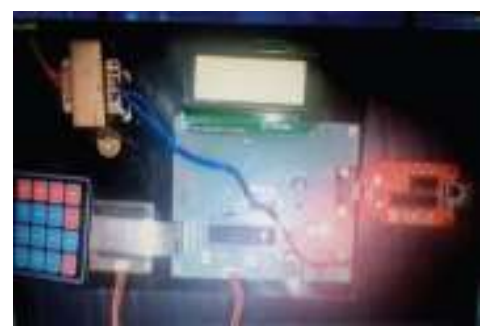

Figure 6 automation in the system

\section{ADVANTAGES AND APPLICATIONS}

Reduction in the time wastage for waiting in long queue lines. Therefore, the time which is required for shopping is minimized.

- Less number of human beings are needed for the service, and by this reduction in the expenditures experienced by the management of the market.

- It keeps updating the buyer about the amount of purchasing so that one can do shopping accordingly.

- Automation in data transferring to counter of billing.

- The system is user friendly.

The automated supermarket system can be used in grocery stores, super markets, shopping malls, clothing showrooms.

\section{CONCLUSION}

An automated system for shopping that uses RFID technology has been implemented successfully. It has been seen by the implementation that the system reduces overall duration of time for shopping for users so the system is user friendly. The goal of the present system is achieved which is to reduce the time consumption. By the implementation of the system there is no need to stand in long queues, the bill will be automatically sent to the billing counter and the buyer has to only pay for purchased products. RFID sensors do not permit to take any product out without scanning the product. Therefore, the system delivers trouble free and user friendly shopping system.

\section{REFERENCES}

1. Z. Ali and R. Sonkusare, "RFID based smart shopping: An overview," in Proceedings - 2014 IEEE International Conference on Advances in Communication and Computing Technologies, ICACACT 2014, 2014.

2. N. Rajabi and A. Hakim, "An intelligent interactive marketing system based-on Internet of Things (IoT)," in Conference Proceedings of 2015 2nd International

Conference on Knowledge-Based Engineering and Innovation, KBEI 2015, 2016.

3. C. Shih, B. C. Liang, C. Z. Lin, N. L. Hsueh, and P. A. Hsiung, "An automatic smart shopping cart deployment framework based on pattern design," in Proceedings of the International Symposium on Consumer Electronics, ISCE, 2011.

4. "RFID Based Automatic Shopping Cart," Int. J. Adv. Sci. Res. Eng., 2018.

5. P. Chandrasekar and T. Sangeetha, "Smart shopping cart with automatic billing system through RFID and ZigBee," in 2014 International Conference on Information Communication and Embedded Systems, ICICES 2014, 2015.

6. Paper on " Smart Shopping Cart for Automated Billing Purpose using Wireless Sensor Networks" by Udita Gangwal, Sanchita Roy, Jyotsna Bapat. 